KYUNGPOOK Math. J. 55(2015), 103-107

http://dx.doi.org/10.5666/KMJ.2015.55.1.103

pISSN 1225-6951 eISSN 0454-8124

(c) Kyungpook Mathematical Journal

\title{
Hyers-Ulam Stability of Pompeiu's Point
}

JinghaO HuANG AND YONGJIN Li*

Department of Mathematics, Sun Yat-Sen University, Guangzhou, 510275 P. R. China

e-mail : hjinghao@mail2.sysu.edu.cn and stslyj@mail.sysu.edu.cn

Abstract. In this paper, we investigate the stability of Pompeiu's points in the sense of Hyers-Ulam.

\section{Introduction}

In 1946, Pompeiu [8] derived a variant of Lagrange's mean value theorem, now known as Pompeiu's mean value theorem.

Definition 1.1. For every real valued function $f$ differentiable on an interval $[\mathrm{a}, \mathrm{b}]$ not containing 0 and for all pairs $x_{1} \neq x_{2}$ in $[a, b]$, there exists a point $\xi$ in $\left(x_{1}, x_{2}\right)$ such that

$$
\frac{x_{1} f\left(x_{2}\right)-x_{2} f\left(x_{1}\right)}{x_{1}-x_{2}}=f(\xi)-\xi f^{\prime}(\xi) \text {. }
$$

Such an intermediate point $\xi$ will be called Pompeiu's point of the function $f$. The geometric meaning of this is that the tangent at the point $(\xi, f(\xi))$ intersects on the $y$-axis at the same point as the secant line connecting the points $\left(x_{1}, f\left(x_{1}\right)\right)$ and $\left(x_{2}, f\left(x_{2}\right)\right)$.

In 1954, Hyers and Ulam [4] considered the stability of differential expressions and proved the following theorem, by which many mathematicians have obtained some interesting theorems.

Theorem 1.2. Let $f: \mathbb{R} \rightarrow \mathbb{R}$ be n-times differentiable in a neighborhood $N$ of the point $\eta$. Suppose that $f^{(n)}(\eta)=0$ and $f^{(n)}(x)$ changes sign at $\eta$. Then, for all $\varepsilon>0$, there exists a $\delta>0$ such that for each function $h: \mathbb{R} \rightarrow \mathbb{R}$ which is $n$-times differentiable in $N$ and satisfies $|h(x)-f(x)|<\delta$ in $N$, there exists a point $\xi$ in $N$ such that $h^{(n)}(\xi)=0$ and $|\xi-\eta|<\varepsilon$.

* Corresponding Author.

Received October 4, 2013; revised October 31, 2013; accepted November 1, 2013.

2010 Mathematics Subject Classification: 34K20, 26D10.

Key words and phrases: Hyers-Ulam stability, Pompeiu's point, Mean value theorem. 
Let $[a, b] \subset \mathbb{R}$ be a closed interval and

$$
\phi=\left\{f:[a, b] \rightarrow \mathbb{R} \mid \mathrm{f} \text { is continuously differentiable, } f^{\prime}(a)=f^{\prime}(b)\right\} .
$$

In 2003, M. Das, T. Riedel and P. K. Sahoo [1] gave a Hyers-Ulam type stability result for Flett's points.

Theorem 1.3. Let $f \in \phi$ and $\eta$ be a Flett's point of $f$ in $(a, b)$. Assume that there is a neighborhood $N$ of $\eta$ in $(a, b)$ such that $\eta$ is the unique Flett's point of $f$ in $N$. Then for each $\varepsilon>0$, there exists $a \delta>0$ such that for every $h \in \phi$ satisfying $h(a)=f(a)$ and $|h(x)-f(x)|<\delta$ for all $x$ in $N$, there exists a point $\xi \in N$ such that $\xi$ is a Flett's point of $h$ and $|\xi-\eta|<\delta$.

Unfortunately, there are some errors in the proof of M. Das et al.. In 2009, W. Lee, S. Xu and F. Ye [6] constructed a counter example to show that theorem is incorrect, then they proved the Hyers-Ulam stability of the Sahoo-Riedel's point, and as a corollary they got the correct theorem of the stability of Flett's point.

Theorem 1.4. Let $f, h:[a, b] \rightarrow \mathbb{R}$ be differentiable and $\eta$ be a Sahoo-Riedel's point of $f$ in $(a, b)$. If $f$ has 2 nd derivative at $\eta$ and

$$
f^{\prime \prime}(\eta)(\eta-a)-2 f^{\prime}(\eta)+\frac{2(f(\eta)-f(a))}{\eta-a} \neq 0,
$$

then corresponding to any $\varepsilon>0$ and any neighborhood $N \subset(a, b)$ of $\eta$, there exists $a \delta>0$ such that for every $h$ satisfying $|h(x)-h(a)-(f(x)-f(a))|<\delta$ for $x$ in $N$ and $h^{\prime}(b)-h^{\prime}(a)=f^{\prime}(b)-f^{\prime}(a)$, there exists a point $\xi \in N$ such that $\xi$ is a Sahoo-Riedel's point of $h$ and $|\xi-\eta|<\varepsilon$.

In 2010, P. Gǎvruţă, S.-M. Jung and Y. Li [3] investigated the stability of the Lagrange's mean value points.

Theorem 1.5. Let $a, b, \eta$ be real numbers satisfying $a<\eta<b$. Assume that $f: \mathbb{R} \rightarrow \mathbb{R}$ is a twice continuously differentiable function and $\eta$ is the unique Lagrange's mean value point of $f$ in an open interval $(a, b)$ and moreover that $f^{\prime \prime}(\eta) \neq 0$. Suppose $g: \mathbb{R} \rightarrow \mathbb{R}$ is a differentiable function. Then, for a given $\varepsilon>0$, there exists a $\eta>0$ such that if $|f(x)-g(x)|<\eta$ for all $x \in[a, b]$, then there is a Lagrange's mean value point $\xi \in(a, b)$ of $g$ with $|\xi-\eta|<\varepsilon$.

In this paper, we prove the Hyers-Ulam stability of Pompeiu's point by employing the ideas of theorem 1.3, 1.4 and 1.5.

\section{Hyers-Ulam Stability of Pompeiu's Point}

In this section, we investigate the stability of the Pompeiu's point.

Theorem 2.1. Let $f, h:[a, b] \rightarrow \mathbb{R}$ be differentiable and $\eta$ be a Pompeiu's point of $f$. If $f$ has 2nd derivative at $\eta$ with

$$
f^{\prime \prime}(\eta) \neq 0
$$


then corresponding to any $\varepsilon>0$, there exists a $\delta>0$ such that for every $h$ satisfying $|h(t)-f(t)|<\delta$ for all $t \in[a, b]$, there exists a point $\xi \in(a, b)$ such that $\xi$ is a Pompeiu's point of $h$ with $|\xi-\eta|<\varepsilon$.

Proof. Without loss of generality, we shall assume that $a, b>0$. Define a real valued function $F$ on the interval $\left[\frac{1}{b}, \frac{1}{a}\right]$ by

$$
F(t)=t f\left(\frac{1}{t}\right)
$$

Since $f$ is differentiable on $[a, b]$ and 0 is not in $[a, b]$, we see that $F$ is differentiable on $\left(\frac{1}{b}, \frac{1}{a}\right)$ and

$$
F^{\prime}(t)=f\left(\frac{1}{t}\right)-\frac{1}{t} f^{\prime}\left(\frac{1}{t}\right) .
$$

Consider the auxiliary function $G_{F}(t):\left[\frac{1}{b}, \frac{1}{a}\right] \rightarrow \mathbb{R}$ corresponding to $F$ defined by

$$
G_{F}(t)=F(t)-\frac{F\left(\frac{1}{b}\right)-F\left(\frac{1}{a}\right)}{\frac{1}{b}-\frac{1}{a}}\left(t-\frac{1}{a}\right) .
$$

Since $\eta$ is a Pompeiu's point, we have

$$
G_{F}^{\prime}\left(\frac{1}{\eta}\right)=F^{\prime}\left(\frac{1}{\eta}\right)-\frac{F\left(\frac{1}{b}\right)-F\left(\frac{1}{a}\right)}{\frac{1}{b}-\frac{1}{a}}=f(\eta)-\eta f^{\prime}(\eta)-\frac{a f(b)-b f(a)}{a-b}=0 .
$$

Moreover, by the assumption that $f^{\prime \prime}(\eta) \neq 0$, we obtain that

$$
G_{F}^{\prime \prime}\left(\frac{1}{\eta}\right)=F^{\prime \prime}\left(\frac{1}{\eta}\right)=\eta^{3} f^{\prime \prime}(\eta) \neq 0,
$$

which implies $G_{F}^{\prime}(t)$ changes sign at $\frac{1}{\eta}$.

According to theorem 1.2, for all $\varepsilon>0$, there exists a $\delta>0$ such that for each function $\phi:\left[\frac{1}{b}, \frac{1}{a}\right] \rightarrow \mathbb{R}$ which is differentiable in $\left(\frac{1}{b}, \frac{1}{a}\right)$ and satisfies $\left|\phi(t)-G_{F}(t)\right|<$ $\frac{3 \delta}{a}$ in $\left[\frac{1}{b}, \frac{1}{a}\right]$, there exists a point $\xi_{0}$ in $\left(\frac{1}{b}, \frac{1}{a}\right)$ such that $\phi^{\prime}\left(\xi_{0}\right)=0$ and $\left|\xi_{0}-\frac{1}{\eta}\right|<\frac{1}{b^{2}} \varepsilon$.

Now, let us define differentiable functions $H$ and $G_{H}$ by

$$
H(t)=\operatorname{th}\left(\frac{1}{t}\right)
$$

and

$$
G_{H}(t)=H(t)-\frac{H\left(\frac{1}{b}\right)-H\left(\frac{1}{a}\right)}{\frac{1}{b}-\frac{1}{a}}\left(t-\frac{1}{a}\right) .
$$

Recall $F(t)=t f\left(\frac{1}{t}\right)$, we have

$$
|H(t)-F(t)|=\left|\operatorname{th}\left(\frac{1}{t}\right)-t f\left(\frac{1}{t}\right)\right| \leq \frac{1}{a}\left|h\left(\frac{1}{t}\right)-f\left(\frac{1}{t}\right)\right|
$$


for all $t \in\left[\frac{1}{b}, \frac{1}{a}\right]$. On the other hand,

$$
\begin{aligned}
\left|G_{H}(t)-G_{F}(t)\right| & \leq\left|H(t)-\frac{H\left(\frac{1}{b}\right)-H\left(\frac{1}{a}\right)}{\frac{1}{b}-\frac{1}{a}}\left(t-\frac{1}{a}\right)-\left(F(t)-\frac{F\left(\frac{1}{b}\right)-F\left(\frac{1}{a}\right)}{\frac{1}{b}-\frac{1}{a}}\left(t-\frac{1}{a}\right)\right)\right| \\
& \leq|H(t)-F(t)|+\left|\left(t-\frac{1}{a}\right)\left(\frac{H\left(\frac{1}{b}\right)-F\left(\frac{1}{b}\right)}{\frac{1}{b}-\frac{1}{a}}\right)\right|+\left|\left(t-\frac{1}{a}\right)\left(\frac{H\left(\frac{1}{a}\right)-F\left(\frac{1}{a}\right)}{\frac{1}{b}-\frac{1}{a}}\right)\right| \\
& \leq|H(t)-F(t)|+\left|\left(\frac{1}{b}-\frac{1}{a}\right)\left(\frac{H\left(\frac{1}{b}\right)-F\left(\frac{1}{b}\right)}{\frac{1}{b}-\frac{1}{a}}\right)\right|+\left|\left(\frac{1}{b}-\frac{1}{a}\right)\left(\frac{H\left(\frac{1}{a}\right)-F\left(\frac{1}{a}\right)}{\frac{1}{b}-\frac{1}{a}}\right)\right| \\
& \leq|H(t)-F(t)|+\left|H\left(\frac{1}{b}\right)-F\left(\frac{1}{b}\right)\right|+\left|H\left(\frac{1}{a}\right)-F\left(\frac{1}{a}\right)\right|
\end{aligned}
$$

for all $t \in\left[\frac{1}{b}, \frac{1}{a}\right]$. Let $|h(t)-f(t)|<\delta$ for all $t \in[a, b]$, we have $\left|G_{H}(t)-G_{F}(t)\right| \leq \frac{3 \delta}{a}$ for all $t \in\left[\frac{1}{b}, \frac{1}{a}\right]$, which implies that there exists a point $\xi_{0}$ in $\left(\frac{1}{b}, \frac{1}{a}\right)$ such that $G_{H}^{\prime}\left(\xi_{0}\right)=0$ and $\left|\xi_{0}-\frac{1}{\eta}\right| \leq \frac{1}{b^{2}} \varepsilon$.

Define $\xi=\frac{1}{\xi_{0}}$. Recall $G_{H}^{\prime}\left(\xi_{0}\right)=0$, which implies $H^{\prime}\left(\xi_{0}\right)=\frac{H\left(\frac{1}{b}\right)-H\left(\frac{1}{a}\right)}{\frac{1}{b}-\frac{1}{a}}$, we obtain that

$$
h(\xi)-\xi h^{\prime}(\xi)=\frac{\frac{1}{b} h(b)-\frac{1}{a} h(a)}{\frac{1}{b}-\frac{1}{a}}=\frac{a h(b)-b h(a)}{a-b},
$$

from which it follows that $\xi$ is a Pompeiu's point of $h$. Moreover,

$$
|\xi-\eta|=\left|\frac{1}{\xi_{0}}-\eta\right|=\left|\frac{\xi_{0}-\frac{1}{\eta}}{\xi_{0} \cdot \frac{1}{\eta}}\right| \leq b^{2}\left|\xi_{0}-\frac{1}{\eta}\right| \leq \varepsilon .
$$

The proof is completed.

Corollary 2.2. Let $f, h:[a, b] \rightarrow \mathbb{R}$ be differentiable and $\eta$ be a Pompeiu's point of $f$. If $f$ has 2 nd derivative at $\eta$ and

$$
f^{\prime \prime}(\eta) \neq 0
$$

then corresponding to any $\varepsilon>0$, there exists a $\delta>0$ such that for every $h$ satisfying $|h(t)-f(t)-c|<\delta$ for $t$ in $[a, b]$, where $c$ is a constant, there exists a point $\xi \in(a, b)$ such that $\xi$ is a Pompeiu's point of $h$ and $|\xi-\eta|<\varepsilon$.

The following counter example shows that Theorem 2.1 will be incorrect if we remove the condition $f^{\prime \prime}(\eta) \neq 0$.

Example 2.3. Let $[a, b]=[1,2]$,

$$
f(x)=0
$$

Then, we can see that $f(x)$ is twice differentiable on $(1,2)$ and $f^{\prime \prime}(x)=0$ for all $x \in(1,2)$. What's more, every $x \in(1,2)$ is a Pompeiu's point of $f(x)$. Let $\eta=\frac{7}{4}$, which is a Pompeiu's point of $f$. 
For sufficiently small $\delta>0$, define

$$
h(x)=\delta\left[4\left(x-\frac{3}{2}\right)^{2}-1\right]
$$

for $x \in[1,2]$. Then, we can know from the geometric meaning of Pompeiu's point that the Pompeiu's point $\xi$ of $h(x)$ is in $\left(1, \frac{3}{2}\right)$, or rather, $\xi=\sqrt{2}$ is the unique Pompeiu's point of $h$ in $[1,2]$.

Finally, we have

$$
|\xi-\eta|=\left|\sqrt{2}-\frac{7}{4}\right|>\frac{1}{4}
$$

In other words, for all $\delta>0$, there exists a twice differentiable function $h$ satisfying $|f-h|<\delta$, but there is no Pompeiu's point of $h$ in the neighborhood of $\eta$ which is a Pompeiu's point of $f$.

Acknowledgements. The authors would like to thank the anonymous referee for his or her suggestions and corrections. This work was supported by the National Natural Science Foundation of China (10871213).

\section{References}

[1] M. Das, T. Riedel, P. K. Sahoo, Hyers-Ulam stability of Flett's points, Appl. Math. Lett., 16(3)(2003), 269-271.

[2] T. M. Flett, A mean value theorem, Math. Gaztte, 42(1958), 38-39.

[3] P. Găvruţă, S.-M. Jung, Y. Li, Hyers-Ulam stability of mean value points, Ann. Funct. Anal., 1(2)(2010), 68-74.

[4] D. H. Hyers, S. M. Ulam, On the stability of differential expressions, Math. Mag., 28(1954), 59-64.

[5] S.-M. Jung, Hyers-Ulam stability of zeros of polynomials, Appl. Math. Lett., 24(2011), $1322-1325$.

[6] W. Lee, S. Xu, F. Ye, Hyers-Ulam stability of Sahoo-Riedel's point, Appl. Math. Lett., 22(2009) 1649-1652.

[7] Y. Li, L. Hua, Hyers-Ulam stability of a polynomial equation, Banach J. Math. Anal., 3(2)(2009), 86-90.

[8] P. K. Sahoo, T. Riedel, Mean Value Theorems and Functional Equations, World Scientific, River Edge, NJ, 1998.

[9] S. M. Ulam, A Collection of the Mathematical Problems, Interscience, New York, 1960. 